vol. 24 - $n^{\circ} 3 \mid 2008$

Numéro ouvert

\title{
La migration au risque de l'illégalité, entre France et Algérie (1998-2004)
}

The Migration at the Illegality's Risk, between France and Algeria (1998-2004)

La migración, a riesgo de la ilegalidad, entre Francia y Argelia (1998-2004)

\section{Marie-Thérèse Têtu}

\section{(2) OpenEdition}

Journals

Édition électronique

URL : https://journals.openedition.org/remi/4831

DOI : 10.4000/remi.4831

ISSN : $1777-5418$

Éditeur

Université de Poitiers

Édition imprimée

Date de publication : 1 décembre 2008

Pagination : 107-127

ISBN : 978-2-911627-50-7

ISSN : 0765-0752

Référence électronique

Marie-Thérèse Têtu, «La migration au risque de l'illégalité, entre France et Algérie (1998-2004) », Revue européenne des migrations internationales [En ligne], vol. 24 - n³ | 2008, mis en ligne le 01 décembre 2011, consulté le 14 avril 2022. URL : http://journals.openedition.org/remi/4831 ; DOI : https://doi.org/ 10.4000/remi.4831 


\title{
La migration au risque de l'illégalité, entre France et Algérie (1998-2004)
}

\author{
Marie-Thérèse TÊTU*
}

À partir d'une approche socio anthropologique, cet article se propose de rendre compte du phénomène des migrations irrégulières algériennes vers la France en se plaçant du point de vue des migrants. Peu de chercheurs ont mené leurs investigations et développé leurs analyses dans cette perspective concernant les migrants ayant réussi à s'introduire à l'intérieur des frontières de l'Europe sans avoir négocié préalablement un statut juridique.

Le phénomène a été étudié dans sa dimension économique et politique. Andréa Réa (2002), Alain Morice (2004) et Emmanuel Terray (1999) ont analysé le lien entre mondialisation économique, ultralibéralisme, déréglementation et migrations et ont vu une concordance des politiques d'immigration, du rôle des États avec des réalités économiques. Emmanuel Terray a en particulier analysé le lien entre migrations clandestines et économie libérale comme un processus de délocalisation sur place. Patrick Weil (1991) a replacé ce phénomène contemporain dans une perspective historique portant sur un demi-siècle de politique française de l'immigration. Catherine Wihtol de Wenden (1999) s'est intéressée à l'histoire et aux enjeux contemporains de la fermeture et de l'ouverture des frontières à l'échelle européenne. D'autres chercheurs (Siméant, 1998 ; Brun, 2006 ; Marin, 2006 ; Vianna, 2006) ont pris pour entrée les liens entre migrations irrégulières et actions collectives au travers de leurs manifestations sociopolitiques. Une autre dimension a été celle des recherches qui ont croisé politique d'immigration et politique de l'asile (Legoux, 1999, 2006), politique d'immigration et politique sociale (Frigoli, 2004) avec ses aspects sociaux et le glissement d'une conception politique de l'asile à une politique humanitaire. Enfin, de nombreux chercheurs ont posé la question du rapport entre le traitement de ces migrations et le respect des droits de l'homme et des procédures juridiques, ainsi que les transformations du monde associatif agissant dans ce domaine (Franguiadakis, Jaillardon et Belkis, 2005).

* Socio-anthropologue, chercheure associée CERS UMR 5193 Université Toulouse Le Mirail, MoDyS UMR 5264 Universités Lyon 2 et Saint-Étienne Jean Monnet. E-mail : mttetu@aol.com. 
Les migrants « irréguliers » y sont essentiellement pris comme des objets de l'actualité, des discours, des opinions publiques, d'une exploitation économique, des acteurs associatifs, du droit, des lois, de l'action sociale, des pratiques administratives ou des politiques d'intégration et d'immigration. Cette recherche se propose de rendre compte de leur expérience, de leur capacité à agir et à prendre des initiatives par des approches compréhensives. Elle s'interroge sur le devenir du migrant acteur et de la migration comme activité (Battegay, 1996). Comment, dans ce cas, se manifeste la production de rapports sociaux originaux, de normes entre ici et là-bas que la notion de territoires circulatoires (Tarrius, 2001) a fait émerger ? Peut-on parler de circulations au sujet des migrations irrégulières et en quels termes ?

\section{LA CIRCULATION SANS LES PAPIERS}

Les dimensions juridiques des États affectent sans aucun doute les capacités des migrants à circuler et faire circuler. L'intention n'est ni de nier, ni de survaloriser les circulations à l'initiative des migrants mais d'examiner leurs formes et leurs sens dans ce cas. Il ne s'agit pas non plus de négliger ou à l'inverse de surestimer les politiques des États-nations. Il est question d'adopter une démarche qui, selon les termes d'Alain Tarrius (2006: 64), prête une attention aux faits d'interaction plus qu'aux approches systémiques. Sans, précise-t-il, "dissocier topiques des circulants et des sédentaires, faits exposés et faits masqués, officialité et subterranéité ».

Les permanences, continuités, adaptations et transformations de l'émigréimmigré, ni d'ici ni de là-bas d'Abdelmalek Sayad, en un circulant et d'ici et de là-bas d'Alain Tarrius peuvent être revisitées à l'aune des routes juridiques qui sont certes imposées mais aussi tracées par les migrants. La qualification par Alain Battegay du migrant comme un acteur et de sa migration comme une activité est confrontée aux limites définies par les politiques des États, aux empêchements au va-et-vient, à "l'obligation de faire face à un problème d'organisation politique des États" (Waldinger, $2006: 31$ ).

La façon dont les migrants y font face fait partie de notre objet d'étude. Dans ce cas, quels sont les savoir-faire mobilisés et élaborés pour "se saisir d'opportunités, mobiliser des ressources et prendre des initiatives socialement significatives " (Battegay, 1996)?

L'intention est ainsi de se situer à la confluence entre l'étude des politiques migratoires et celle des circulations migratoires. Notre objet est donc devenu l'analyse des langages, des pratiques et des itinéraires des migrants irréguliers algériens en interaction avec les procédures juridiques, les catégories établies, les politiques adoptées les concernant et leurs conséquences dans la période 1998-2004 ${ }^{1}$.

1 Une période qui correspond à la mise en place de la procédure de l'asile territorial. Alors que l'interprétation française de la Convention de Genève réservait l'asile aux demandeurs persécutés par leur État, l'asile territorial, géré par le Ministère de l'Intérieur, créé en 1999, 
Nous avons adopté la vision de migrants qui, bien qu'en situation irrégulière et aspirant à obtenir un statut, ne sont pas isolés mais au contraire bien présents dans la vie sociale. Nous nous proposons de voir ce qu'une approche en termes de mondes sociaux et de négociation (Strauss, 1999) peut apporter à une compréhension des phénomènes sociaux auxquels ils sont confrontés et qu'ils contribuent à produire. Dans notre cas des négociations ont pu se mener dans divers espaces et à différentes échelles, avec le préfet et ses services, mais également avec un propriétaire immobilier, un employeur ou encore un ou une future conjointe et sa famille. Ces négociations ne se mènent ni dans un vide institutionnel, ni en vase clos, et les contextes de négociation sont à analyser en rapport avec les contextes structurels et les possibilités pour les acteurs de recourir à des options alternatives.

L'objectif des négociations pour les migrants viserait à dépasser l'asymétrie des relations sociales, les rapports de domination, à introduire de la réciprocité, à augmenter la marge de choix et de liberté, à limiter les contraintes autant que possible. Quels sont les diverses modalités, les différents registres et espaces de reconnaissance pour les migrants irréguliers algériens ? En faisant l'hypothèse qu'au-delà des papiers et d'une reconnaissance juridique, les migrants irréguliers viseraient à être reconnus comme des individus capables d'agir et de choisir leur vie.

Pour mener nos investigations nous avons opté pour une description sélective, liée à une expérience personnelle intense et à des relations réciproques dans les «mondes» des sans papiers ${ }^{2}$. Nous avons visé l'exploration et l'interprétation d'un processus mouvant et changeant par la mise en lien et l'analyse de matériaux et de sources aux statuts différents. À partir d'une ville de la Drôme, Romans sur Isère, nous avons suivi dans la durée, trois à quatre ans, 60 Algériens et Algériennes dits sans papiers, dont une dizaine d'assez près, ainsi que les acteurs significatifs avec lesquels ils étaient en contact. Nous avons recueilli des récits, suivi et reconstitué des parcours, noté des conversations, partagé des tranches de vie, des événements et des épreuves dans trois directions qui apparaissaient essentielles : la carrière administrative et ses démarches, la vie ordinaire et ses épreuves, les liens avec l'Algérie.

s'appliquait aux demandeurs lorsque l'agent de persécution ne pouvait être clairement être identifié ou que la menace provenait de groupes armés. La procédure a en fait été mise au point spécifiquement pour les Algériens victimes des islamistes, mais l'ensemble des sans papiers algériens y ont eu largement recours, ce qui les a amené à déposer des dossiers et à fréquenter les préfectures. L'octroi de l'asile territorial n'a atteint que des niveaux dérisoires ( $1,5 \%$ des demandes en 2001 comme en $2003,0,3 \%$ en 2002). La procédure a été supprimée en 2004.

2 La dénomination de sans papiers (un terme inexact, prêtant à confusion, dont la connotation militante est préférée par les associations et les concernés à celle, criminalisante et négative de clandestin) a été popularisée par les associations de soutien et reprise par les médias. Elle fait donc partie du langage courant et recouvre un ensemble de personnes dont les situations juridiques sont différent mais ayant en commun de ne pas être régularisées par un titre de séjour. C'est dans ce sens que nous l'employons dans cet article. Sur le choix, les définitions et les usages des termes désignant les migrants en situation irrégulière voir Didier Fassin (1996). 
Le suivi de leur carrière administrative nous a amené à fréquenter la vie d'une association, des permanences juridiques, les services des préfectures, le tribunal administratif, à rencontrer des élus, un préfet, des avocats, à écrire des récits, à remplir des dossiers, à connaître les lois, les pratiquer, les interpréter, à réfléchir à la façon de les contourner ou de les utiliser, à suivre et participer à des cérémonies de mariage. Elle nous a donné accès pendant l'été 2004, à 1340 dossiers d'étrangers constitués par l'Asti (Association de soutien à tous les immigrés), dont 852 d'Algériens. Il s'agissait de dossiers actifs, en cours.

L'observation de leur vie ordinaire et de leurs épreuves nous a conduite à fréquenter un centre d'hébergement, des squats, les marchés, les exploitations agricoles, les commerces, à participer à des fêtes familiales et religieuses, à des réunions entre amis, à des conversations, à rencontrer des familles ou des cooriginaires en situation régulière en France, un policier municipal évangéliste, un charcutier catholique, des associations humanitaires, des propriétaires immobiliers, des enseignants, des candidates au mariage. La vie ordinaire c'est aussi la découverte d'activités, la pratique d'échanges, la recherche de logements, d'activités lucratives, une exploration de la ville des sans papiers et de ses ressources.

La connaissance des liens avec l'Algérie ce sont les conversations, les liens téléphoniques, les échanges d'informations, les récits de retours des régularisés, de ceux qui arrivent du bled. Puis ce fut un séjour chez quelques familles de sans papiers à Alger et Sidi Bel Abbès, l'accompagnement du retour d'une femme régularisée à Guelma, la rencontre avec deux expulsés, avec des candidats au départ et leurs familles. Une première perception d'aspects de la vie quotidienne dans ce pays et une lecture régulière de la presse algérienne.

Ce sont donc ces trois sources, la carrière administrative et ses démarches, la vie ordinaire et ses épreuves, les liens avec l'Algérie, et leur croisement qui ont constitué des lignes d'investigation et d'analyse. Mon travail n'est certainement pas une description complète de la vie des sans papiers. Ce sont des fragments qui correspondent à une période, un passage, ce sont des situations, des scènes éphémères dans des contextes changeants. Il s'agit d'un objet marqué par l'incertitude et la précarité. Nous sommes face à du provisoire, mais du provisoire qui peut durer et surtout ce provisoire n'est pas qu'une salle d'attente des papiers. Il se passe des choses et c'est une expérience qui compte.

Exposés les uns à la suite des autres, les documents officiels, les rencontres informelles et les séjours en Algérie donnent des éclairages, des informations, des points et des angles de vue différents et complémentaires; des éléments quantitatifs et qualitatifs, objectifs et subjectifs ; des données qui s'emmêlent, s'enchevêtrent, s'encastrent, et qu'il est possible de croiser, de mettre en dialogue, de démêler.

\section{LES PROFILS DES MIGRANTS IRRÉGULIERS ALGÉRIENS}

Nous les avons établis à partir de l'analyse des dossiers en cours déposés par des Algériens à l'Asti Drôme-Ardèche au moment de l'étude, en 2004. Tous les étrangers ne déposent pas un dossier à l'Asti, tous les Algériens en situation irrégulière 
non plus. Cependant, cette association est une adresse, un passage, une ressource qui, à cette période, circulait en particulier entre les Algériens, comme les restos du cœur, le centre d'hébergement, le secours catholique, pour les aider à déposer des dossiers et pour avoir une domiciliation pour la préfecture. Beaucoup y ont eu recours.

\section{Les dossiers : une source de données}

Dans les dossiers officiels, derrière les papiers, par bribes, nous pouvons deviner, imaginer et faire vivre des individus. Des parcours, des discours, des données se répètent et prennent sens. Ces données ont trait aux lieux d'origine des migrants, à leurs sexes et leurs âges, à leurs professions, aux raisons de leurs départs telles qu'ils les ont déclarées. Ces dossiers sont constitués par les militants et bénévoles de l'Asti pour accompagner les migrants dans leurs démarches auprès des administrations.

\section{Des lieux d'origines diversifiés}

Sur 697 dossiers déposés à l'Asti Drôme, les provenances des migrants indiquent 34 villes et 56 villages différents. Le tableau 1 ci-dessous indique les quatre régions d'où proviennent 633 d'entre eux, et pour chacune d'entre elles des localités dont proviennent au moins quatre Algériens ayant déposé un dossier.

Tableau 1 : Principales régions et villes d'origine des Algériens ayant déposé un dossier à l'ASTI Drôme-Ardèche, 2004

\begin{tabular}{|c|c|c|c|c|c|c|c|}
\hline ORANAIS & 169 & CHELIF & 169 & ANNABA & 136 & ALGEROIS & 72 \\
\hline dont & & dont & & dont & & dont & \\
\hline $\begin{array}{l}\text { Sidi Bel } \\
\text { Abbès }\end{array}$ & 60 & $\begin{array}{l}\text { Chleff et } \\
\text { villages }\end{array}$ & 128 & $\begin{array}{l}\text { Guelma et } \\
\text { villages }\end{array}$ & 93 & Alger & 38 \\
\hline Oran & 47 & $\begin{array}{l}\text { Rélizane et } \\
\text { villages }\end{array}$ & 41 & Annaba & 26 & $\begin{array}{l}\text { Blida et } \\
\text { environs }\end{array}$ & 12 \\
\hline Mostaghanem & 29 & & & Skikda & 10 & Tipaza & 8 \\
\hline Mascara & 16 & & & Souk Ahras & 5 & $\begin{array}{l}\text { Médéa et } \\
\text { environs }\end{array}$ & 5 \\
\hline Tlemcen & 8 & & & & & Cherchell & 3 \\
\hline $\begin{array}{l}\text { Aïn } \\
\text { Temouchent }\end{array}$ & 4 & & & & & Bouira & 3 \\
\hline
\end{tabular}

Source : Dossiers en cours en 2004.

La grande majorité d'entre eux (près de quatre sur cinq) seraient d'origine urbaine. Cette forte proportion doit être pondérée car, pour une partie des dossiers, le lieu d'origine n'a pas été établi à partir du passeport; ils peuvent donc avoir indiqué le nom de la ville la plus proche de leur village. Mais il s'agit d'une migration majoritairement urbaine, et ce caractère est accentué par les caractéristiques d'un certain nombre de villages, qui sont en fait des gros bourgs ou des zones d'habitation périphériques à la ville. 
Les Algériens rencontrés présentent leur migration comme une entreprise individuelle ou familiale, où chacun se débrouille et choisit sa voie. Cependant, le caractère individuel affirmé par les migrants doit être modéré. La présence de cooriginaires est un appui pour au moins un tiers des dossiers, même si cette aide ne peut se prolonger longtemps. Ainsi, sur un total de 697 dossiers, 128 personnes viennent de Chleff et 93 de Guelma, deux villes dont sont originaires des familles déjà installées à Valence et à Romans.

\section{Une majorité d'hommes jeunes}

La plupart des migrants (près de $80 \%$ ) sont des hommes. Parmi eux tous les âges de 0 à plus de 60 ans sont représentés, mais la majorité d'entre eux ont un âge compris entre 20 et 35 ans avec un pic de 26 à 30 ans. Les femmes, très minoritaires, sont plus âgées ; le plus grand nombre a entre 36 et 40 ans. Certaines arrivent en famille, avec mari et enfants, mais d'autres, femmes divorcées ou encore célibataires à cet âge avancé, sont parties seules

\section{Professions en Algérie avant le départ}

Les professions mentionnées sur les dossiers ne sont que des indications. On note d'abord la faible représentation des chômeurs, à peine $6 \%$, alors que les statistiques officielles algériennes évaluaient globalement à $15 \%$ leur pourcentage dans le pays en 2005. Enfin la profession retenue est souvent celle qui se trouve sur le passeport. Beaucoup des migrants ont exercé tour à tour plusieurs métiers : ils peuvent avoir été photographe et coiffeur, ingénieur et chauffeur de taxi, des métiers sans lien évident. Ils choisissent celui qui leur paraît améliorer leur profil.

Figure 1 : Professions exercées en Algérie

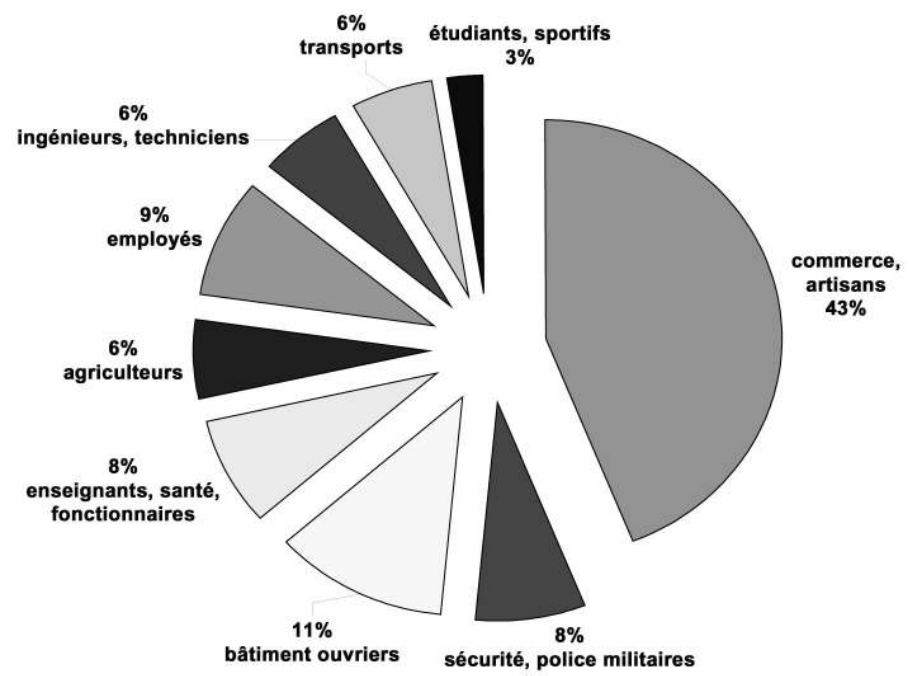

Source : 653 dossiers d'Algériens en cours en 2004 - Asti Drôme-Ardèche. 
On notera parmi eux une forte représentation des métiers du commerce et de l'artisanat (43\%), particulièrement chez les jeunes, exercés souvent dans le secteur de l'économie informelle ${ }^{3}$. Une activité vers laquelle se tourne une grande partie des Algériens qui ne trouvent pas d'emplois salariés. Viennent ensuite les ouvriers $(11 \%)$. Mais les salariés du secteur public, personnels de la police et de la sécurité $(8 \%)$, enseignants, personnels de la santé et fonctionnaires (8\%) sont bien représentés. Enfin la part des ingénieurs $(6 \%)$ est aussi élevée que celle des agriculteurs $(6 \%)$. Le fait que nous soyons dans la Drôme peut conduire à ce que certaines professions soient éventuellement moins représentées, en particulier les étudiants ou professions intellectuelles. Mais ce n'est pas sûr, quelles que soient leurs professions antérieures les sans papiers se trouvent en France soumis à la même condition et cherchent des revenus là où ils peuvent espérer en trouver.

Les salaires, indiqués sur les fiches de paie jointes aux dossiers, donnent des indications à la fois sur la faiblesse des revenus et sur l'échelle de ces revenus selon les professions. En 2002, le smic algérien s'élevait à 8000 dinars par mois (à peu près 80 euros). Les salaires variaient de 7260 dinars pour une vendeuse en pharmacie à 15000 dinars pour un policier des brigades spéciales, un employé de banque et un chauffeur livreur. Un ingénieur percevait 11400 dinars, un instituteur ayant 10 ans d'ancienneté 13000 dinars, tandis qu'un mécanicien gagnait 13000 dinars et un soudeur 12385 da. Enfin, les veuves, dont le mari a été assassiné, percevaient une pension de 5700 da.

\section{Des itinéraires traditionnels et diversifiés}

Quelques indications sur les itinéraires peuvent être relevées dans les dossiers à partir des visas apposés sur les passeports, des préfectures où ont été déposés les dossiers, des récits, des adresses officiellement déclarées et des contacts officieux donnés à l'association, des notes en marge du dossier officiel. Les circulations en France et en Europe ne sont pas toujours mentionnées, la présence de la famille non plus, et il y a souvent un écart entre l'adresse déclarée et l'adresse réelle. Dans la majorité des cas, un numéro de portable est donné comme le moyen le plus sûr pour les joindre.

Il ressort que le plus grand nombre arrive par bateau ou par avion en possession d'un visa. La majorité d'entre eux ont de la famille en France, qui leur sert de base d'atterrissage, puis de boîte postale. Car cet accueil ne dure jamais longtemps. Pour les jeunes célibataires, mais aussi pour certaines familles, on peut noter plusieurs changements d'adresses, de villes, qui laissent à penser que dans un premier temps ils cherchent où s'installer. Ils rejoignent alors la Drôme, soit pour se rapprocher de leur famille ou de regroupements de gens originaires de leur ville, ou bien parce que d'autres sans papiers les ont informés des possibilités de travail dans cette région.

3 En 2003, le Centre national économique et social évaluait l'emploi informel à 1249000 personnes $(17,2 \%$ de l'emploi total) et selon le ministère du commerce, l'informel représentait $40 \%$ de la sphère commerciale où exerceraient 850000 commerçants. Source : El Watan du $24 / 10 / 2004$. 
Parmi ceux qui n'arrivent pas directement d'Algérie plusieurs cas de figure sont à noter. Les uns ont fait des allers et retours entre la France et l'Algérie, pour comme ils me le diront "tester le mouvement » avant de se décider à quitter leur pays de façon plus durable. D'autres, avant de venir en France, ont voyagé, fait des tentatives vers d'autres destinations (Libye, Syrie, Turquie, Espagne en particulier). D'autres encore, ont fait des détours selon les opportunités ou les visas qu'ils parvenaient à obtenir. Quelques parcours reconstitués à partir des passeports, des visas et des dossiers illustrent cette labilité des destinations, des statuts, et des projets.

\section{Les raisons officielles et officieuses de leur départ}

Dans les dossiers et en particulier dans les récits qui doivent être rédigés pour les demandes d'asile peuvent se lire les raisons officielles du départ et en filigrane se devinent des raisons plus profondes, inavouées ou inavouables, complexes et imbriquées. Les stéréotypes que reprennent les Algériens, les faits qu'ils racontent et dont ils n'ont peut être pas été eux-mêmes victimes, les preuves qu'ils produisent, ne sont pas choisis par hasard, témoignent d'un contexte, de conditions de vie, de situations et de leurs perceptions par les individus. On peut y lire des échos de la crise sécuritaire, politique et économique traversée par l'Algérie dès les années 1985 et de la période de terrorisme qu'a connu ce pays depuis 1991 à partir de l'interruption du processus électoral et du recours à la violence.

La violence et l'insécurité subies directement ou indirectement sont les raisons les plus souvent évoquées. Au-delà des stéréotypes auxquels ont recours le plus couramment les migrants parce qu'ils pensent qu'ils sont recevables par les autorités françaises, le racket, les faux barrages et les lettres de menaces des groupes terroristes, d'autres indices donnent une idée des violences, de la nature de l'insécurité et de leurs conséquences associées.

Une perte de confiance généralisée s'exprime dans ces courriers et est encore plus durement ressentie et difficile à évoquer quand elle s'est rapprochée du voisinage jusqu'à entrer au cœur des familles. Quelques récits, après des généralités sur le climat de violence et d'insécurité, donnent des détails qui indiquent que la famille entière est touchée par les agissements de certains de ses membres ou qu'elle est divisée. La proximité peut aussi se transformer en hostilité, et sont alors évoqués le fait que tout le monde se connaît et les règlements de compte. Le voisin de toujours devient un suspect, un ennemi potentiel.

Les conséquences de cette insécurité se cumulent aux difficultés économiques déjà existantes ou qu'elles renforcent, et conduisent un peu plus à l'éclatement des familles et des solidarités familiales qui ne peuvent plus ou ne veulent plus faire face. Il n'est pas rare non plus que ce soit un faisceau de raisons qui, cumulées, justifient la décision de partir. Il leur est possible alors de mettre sur le même plan trois ans de chômage et un frère gendarme qui travaillait pour les terroristes. Ou encore l'incendie de la maison, l'exode vers la ville et un dossier de création d'une micro entreprise qui n'aboutit pas auprès des banques et des administrations. La destruction d'outils de 
travail de l'entreprise d'un ingénieur est associée aux violences de la gendarmerie et à son divorce ; Le jeune dont les parents divorcent, sa convocation pour le service national et sa mise à la porte par son père après son remariage.

Les départs suite à un divorce des parents, ou à son propre divorce sont relativement courants. Le manque de logements aggrave les problèmes de cohabitation, entraîne des mises à la porte par la famille, par le père, par le mari, par la belle famille, par les frères, par les enfants, et ces situations angoissantes transpirent dans les récits. En particulier pour les femmes et les veuves.

Les revenus, l'argent, le vol, l'atteinte aux biens privés mais aussi la corruption semblent faire partie des pratiques ordinaires. Les récits emploient beaucoup l'argument du racket. S'il est loin d'être avéré dans de nombreux cas, et s'il est une formule passe-partout qui circule entre les migrants, son emploi témoigne tout de même qu'il paraît plausible pour les Algériens. Ils savent, ils ont entendu dire autour d'eux que ceux qui avaient des biens, de l'argent, un commerce n'étaient pas à l'abri de se faire déposséder par des inconnus mais aussi par des employés de l'État. Les faillites, les spoliations, l'absence de respect de la loi mais aussi de protection juridique transparaît dans les courriers et les dossiers. Le vol d'un taxi, l'incendie d'un garage, d'une maison, la demande de produits de l'entreprise où une personne travaille, les faillites ou fermetures rapides d'une affaire, l'intrusion dans les domiciles privés sont autant d'exemples plus précis qui émaillent les récits.

Face à toutes ces raisons qui poussent au départ, à cette situation qui n'a cessé de se dégrader, aux relations familiales et de voisinage mises à mal, l'État, ses institutions, les diplômes obtenus, l'éducation reçue ne semblent plus d'aucuns secours et ne garantissent plus d'un meilleur sort. Ce dont témoignent à mots couverts ceux qui en Algérie avaient un diplôme, un métier, un emploi, une situation ou parfois une carrière brusquement interrompue.

À partir de cette source complétée et épaissie par des conversations informelles menées en direct avec les migrants nous pouvons déjà avoir une idée plus précise des raisons de cette migration et des profils des individus concernés.

Il s'agit d'une migration urbaine, qui ne concerne pas des régions particulières mais s'étend à toutes les villes du pays. Les célibataires partent seuls. Les familles partent souvent au grand complet, le couple et les enfants. L'entrée en France se fait majoritairement de façon légale et s'appuie sur des opportunités multiples, présence de la famille ou d'amis, obtention ou possibilité d'achat d'un visa, connaissance d'une possibilité juridique de régularisation (naissance, mariage, demande d'asile). Enfin, une part non négligeable des migrants atteste d'un retour en France après le retour au bled, ou d'une proximité avec des personnes de la famille qui vivent ou ont vécu en France. Cette proximité, ajoutée aux images et aux informations en provenance de ce pays par la télévision, fait que la France c'est déjà un peu chez eux.

Cette migration touche toutes les tranches d'âge mais majoritairement les jeunes. Les femmes seules ou en couple occupent une place significative dans cette migration $(22 \%)$ et sont en moyenne plus âgée. Un âge qui semble correspondre pour 
une part d'entre elles avec un divorce ou une difficulté à se marier en Algérie. Des contacts en Algérie semblent montrer que des familles commencent à envisager, voire à encourager, le départ de jeunes femmes célibataires. Les « mis à la retraite » anticipée partent aussi pour améliorer leurs revenus et s'occuper.

Une grande diversité de milieux sociaux se retrouve dans cette migration. Concernant les emplois et professions exercées, les individus se déclarant commerçants seraient les plus nombreux. Les victimes de la privatisation et de la désétatisation brutale sont aussi très présentes dans les dossiers entraînant des ruptures de carrière et des déclassements professionnels, économiques et symboliques. Les agents de l'État ou les professions liés au secteur public sont également représentés. Ils se perçoivent comme des cibles potentielles de la violence sans bénéficier des avantages liés à cette fonction, en particulier sur la question des revenus, des évolutions de carrière, de la reconnaissance de la profession et de sa valorisation. De façon plus générale, le travail est une denrée rare, qui ne donne ni accès à des droits comme le logement, ni accès à des biens ou des revenus jugés suffisants.

Dans le même temps, la télévision, la présence inaccessible de biens de consommation avec le passage à l'économie de marché donnent une connaissance et créent une envie de ces mêmes biens et d'autres modes de vie.

L'absence de logements, la promiscuité que doivent supporter les individus et qui empêche l'autonomie et la réalisation d'aspirations individuelles a des conséquences fortes et directes sur la dé-liaison et l'éclatement des familles. Ce dont témoignent les cas qui signalent un départ suite à un divorce ou une « mise à la porte». Une situation que l'obtention d'un travail ne suffit pas à améliorer. Elle mine, envenime les rapports familiaux et est sans doute une des causes des divorces. Le logement, son exiguïté et ses problèmes de cohabitation, illustre bien le temps et l'énergie consacrés en particulier par les femmes à la gestion des soucis quotidiens.

La violence, l'insécurité sont devenues insidieuses, proches et quotidiennes. Cumulées à des difficultés économiques qu'elles ont pu aussi engendrées, et à une perte de confiance dans l'État, elles mettent les familles, les valeurs et les solidarités familiales, à rude épreuve. Les individus, tout en voulant vivre en personnes libres, se retournent vers les familles qui ne peuvent plus répondre à ces demandes.

La migration serait alors perçue comme un des modes de résolution de la contradiction. Quatre profils sont apparus les plus concernés et par cette contradiction, et par la migration irrégulière comme mode de résolution.

\section{Quatre profils de migrants algériens irréguliers}

Les migrations illégales concerneraient non pas des catégories très précises de la société, des origines géographiques bien délimitées, mais plutôt des profils qui correspondraient à des familles et des individus qui se sentiraient les plus touchés, qui disposeraient de moyens ou de ressources facilitant le départ, et qui adhéreraient à la perspective que la vie est meilleure ailleurs. 


\section{Les jeunes en quête d'avenir}

Les jeunes sont les plus nombreux dans cette situation ${ }^{4}$, en particulier les jeunes garçons pour qui, à la différence des filles, les études et les diplômes ne sont plus des moyens de valorisation personnelle reconnus par la société. Les trabendistes ${ }^{5}$, qui gagnent de l'argent et qui voyagent, ont meilleure réputation qu'eux. Ils se sentent coincés, dans une situation sans issue, ni perspective. Ils vivent au plus près de la violence et cherchent les moyens d'en sortir. Ils veulent une place dans une société qui ne leur en fait pas. Ils ont peur de l'avenir. Ils aspirent à une démocratisation, à des libertés individuelles, auxquelles ils ne croient plus. Perçus comme un «facteur oscillant », une « masse flottante » (Djerbal, 2001), ils inquiètent les adultes, y compris leurs proches Les jeunes rencontrés semblent hésiter entre aspirations individuelles et sécurité de la famille, entre exigence de droits sociaux et réussite rapide, individuelle autour des valeurs attribuées à l'argent et aux biens de consommation, entre reconnaissance par les leurs et fuite vers l'ailleurs. La migration est perçue comme un voyage initiatique, un défi, un modèle de réussite et de valorisation. Il faut partir pour réussir, pour devenir quelqu'un et être un individu reconnu. Que ce soit de façon illégale n'est au départ que de peu d'importance. L'enjeu est tel que seul importe d'échapper à l'expulsion, le signe absolu de l'échec, qui signifierait une mort symbolique.

\section{Les femmes en quête de mari et d'autonomie}

Les femmes en situation irrégulière sont minoritaires, mais représentent tout de même $22 \%$ des Algériens ayant déposé un dossier à l'Asti. La majorité d'entre elles ont entre 21 et 40 ans. Elles peuvent être célibataires, mariées ou divorcées. Elles viennent souvent chercher un mari dans des conditions qui leur conviennent mieux que ceux qu'elles pourraient épouser en Algérie. Certaines sont prêtes à accepter beaucoup de choses pour pouvoir se marier et aussi pour avoir des papiers. Des femmes viennent également chercher un mari à distance de la famille et de ses obligations. Alors que d'autres encore, mariées en Algérie, profitent de leur départ pour se mettre à distance d'un mari ou d'une famille non choisis, mal aimés ou maltraitants. Des femmes divorcées viennent dans un pays où il leur semblera plus facile de vivre sous ce statut, ou parce que plus rien ne les retient pour s'assumer comme femme seule et indépendante. Enfin, chez les jeunes femmes, on retrouve tous les cas de figure, celles qui viennent d'abord pour les papiers et sont prêtes à prendre l'époux qu'elles trouvent ou qu'on leur trouve, quitte à disparaître une fois les papiers obtenus. Ou encore celles qui viennent pour pouvoir choisir plus librement leur mari, ou pour échapper au mariage à tout prix. Ce sont des aspirations à une vie plus autonomes qui guident alors le projet migratoire. Les conditions de la réussite de leur recherche sont liées à des atouts acquis en Algérie, les diplômes, mais peut être plus encore une expérience de l'autonomie,

4 En 2004, $67 \%$ des Algériens avaient moins de 29 ans (source : office nationale des statistiques) et en 2005, ils représentaient $75 \%$ des chômeurs.

5 Appellation issue du mot contrebande donnée à ceux qui font du commerce en voyageant et en faisant passer des produits d'un pays à un autre. 
d'une activité économique et professionnelle, des réseaux familiaux ou la capacité à s'en créer d'autres. Néanmoins, on peut retenir que la diversité de leurs aspirations, de leur mode de vie, de leurs parcours reflète sans doute une réalité algérienne contrastée, mais pointe autant des blocages de la situation des femmes que l'émergence de nouvelles pratiques sociales féminines dans la société d'origine.

L'élévation de l'âge du mariage et la difficulté à se marier au pays sont attestés $^{6}$, ainsi que la volonté des femmes d'émerger dans la vie publique, sociale et professionnelle avant même la migration. La migration, plus qu'une rupture, est une poursuite dans d'autres conditions et contextes d'un processus déjà engagé avec le recours à des compétences et des pratiques acquises et expérimentées auparavant. L'investissement des femmes dans la vie publique et sociale en migration est patent. Des femmes sans papiers trouvent comme les hommes des emplois non déclarés, au noir. D'autres poursuivent et adaptent des activités professionnelles acquises au pays, comme la coiffure à domicile, ou la couture. Elles font la démonstration d'une finesse et d'une grande pugnacité dans leurs démarches auprès des administrations ou des institutions. Il n'est pas rare que dans les couples les papiers soient l'affaire des femmes. Quelles que soient leurs tenues vestimentaires, très variables, qu'elles aient renoncé ou non au port du Hidjab avec la traversée de la mer, elles sont présentes et visibles dans l'espace public.

\section{Les déclassés}

D’autres sans papiers ont été les sacrifiés, les déclassés de la privatisation ${ }^{7}$, ou ont été bernés par l'État. Les uns ont été licenciés et ont perdu au-delà de leur emploi leur statut social. Les autres, en plus petit nombre, ont accepté de reprendre sans les moyens nécessaires leur entreprise privatisée, qui faisait souvent partie des canards boiteux des entreprises nationales privatisées. Cette reprise des anciennes entreprises nationales par quelques uns de leurs anciens salariés a également permis que les licenciements des autres salariés s'opèrent sans opposition.

Beaucoup de salariés des entreprises publiques âgés de 50 ans ont été mis en préretraite. Leur licenciement leur laisse du temps libre, et leurs indemnités ne suffisent pas à vivre correctement. Un certain nombre d'entre eux sont disponibles pour la migration, même illégale, à la fois pour s'occuper et pour améliorer leurs revenus. Ils viennent se constituer par eux-mêmes une sorte de « retraite complémentaire ».

6 Selon une enquête du ministère de la santé en 2003 le célibat augmente en Algérie et l'âge moyen au mariage ne cesse de reculer pour les hommes (33 ans) comme pour les femmes (29,6 ans) (CREAD 2004).

7 L'édition El Watan du 26/05/2005 rapporte les propos du premier ministre Ahmed Ouyahia confirmant à l'Assemblée nationale la dissolution entre 1996 et 1998 de 1000 entreprises et la suppression de 400000 emplois (pour une population active de 7,2 millions de personnes en 2002). 
Les préretraités licenciés, maltraités par l'État, peuvent aussi se sentir délaissés ou rejetés par la famille. Ils ressentent alors de façon accrue les inégalités de revenus, leur statut social au sein de la famille, leur situation de divorcée quand ce sont des femmes. Ils reprochent à la société algérienne le recul des valeurs familiales au profit de l'argent. Ils peuvent aussi revendiquer et exercer leur autonomie, leur indépendance, leur liberté tout en sollicitant le soutien de la famille ou de certains de ses membres. Ils peuvent être prêts à tout, à l'illégalité, à l'expulsion, à bousculer les réputations pour réussir à gagner de l'argent pour rentrer « la tête haute ».

\section{Les « revenus » du retour}

Les cas de sans papiers qui ont vécu en France, sont retournés en Algérie et reviennent par la petite porte sont très courants et correspondent à plusieurs cas de figures. Il peut s'agir de femmes qui à l'adolescence ont été mariées en Algérie. Quelques unes reviennent seules, avec ou sans les enfants, parfois après un divorce. Mais la plupart de celles que j'ai rencontré reviennent avec mari et enfants, et ce sont souvent elles qui sont à l'initiative du départ et qui, une fois en France, prennent les affaires en main. Elles ont souvent en France des parents, des frères et sœurs avec lesquels elles s'entendent plus ou moins bien, et qui leur servent plus ou moins d'appui. Un événement de trop en Algérie a pu déclencher leur décision. Ou bien, les difficultés s'accumulant, l'absence d'avenir persistant, elles ont pu juger qu'avec ce qu'elles connaissaient du pays de leur enfance ou les souvenirs qu'elles en avaient gardé, c'en était trop et que leur famille s'en sortirait mieux grâce à leur retour. D'autres femmes avaient pu choisir d'elles mêmes d'aller se marier au bled. Souvent, c'est un divorce qui leur fait estimer qu'elles n'ont plus rien à faire là-bas, et qu'elles assumeront mieux leur condition de femme divorcée en France.

J'ai également rencontré des hommes qui ont grandi en France et qui sont allés en Algérie pour suivre leurs parents. Leur retour en France, plus que leur amour ou la nostalgie du pays de leur enfance, est souvent motivé par la situation en Algérie qui les a contraints à se dire qu'il n'y avait plus rien à faire là-bas. Si la situation s'améliorait, ils se poseraient sans doute à nouveau la question de la réinstallation en Algérie, mais en préparant soigneusement ce nouveau déplacement : logement et emploi, mais surtout garantie de pouvoir repartir à nouveau si la situation venait à se dégrader.

Enfin, quelques cas riches d'enseignements sont ceux de jeunes adultes qui avaient pris d'eux-mêmes la décision de quitter la France pour aller faire leur vie en Algérie. Ce sont ceux que j'appelle les « revenus du retour ». Ils partagent une expérience et une connaissance et d'ici et de là-bas. Parce qu'ils ont fait le retour à l'âge adulte, de façon volontaire et à leur propre initiative, on peut les créditer d'une qualité de jugement dénuée d'illusions, libérée du mythe du paradis français ou de celui du bled imaginaire. À leur place, avec leur expérience singulière, dans un contexte particulier, ils n'ont vécu ni la France rêvée, ni l'Algérie inventée mais les deux pays en direct et en clair. Ce ne serait pas eux qui seraient ni d'ici, ni de là-bas, dans une double absence, mais les sociétés qui les mettraient toujours dans la peau de l'immigré. À l'image de ce que m'a raconté Mahdi, 45 ans : "Là-bas j'ai travaillé dans une entreprise de transports publics. J'avais un accent, j'étais l'immigré. Un jour 
il y a une vieille femme qui m'a regardé comme si j’étais un fou et qui a dit : "il a laissé le pays du paradis pour venir dans le pays de la misère..." Le choix de la nationalité, je crois moi, c'est un choix de mode de vie... Avec la fermeture des frontières on se sent prisonnier... Le gars qui a des papiers on dit qu'il est libéré. Je veux pouvoir voyager et revenir, comme tout le monde ». Il était parti de France suite à un licenciement économique en 1986 avec le projet d'ouvrir un garage en Algérie qui n'a pu aboutir et il est revenu en France en 2003. Une employée de la préfecture lui aurait dit que ceux qui partent et reviennent seraient considérés comme des déserteurs.

\section{LES FIGURES DU HARRAGA ET DU SANS PAPIER}

Quels que soient les profils au départ, une partie des candidats à la migration n'a pas d'autres choix que de prendre la voie illégale. Certains le font délibérément, plus ou moins en connaissance de cause. Ils se reconnaissent plutôt dans la figure du harraga $^{8}$. D'autres prennent le risque, dans l'espoir de régulariser au plus vite leur situation. Ils préfèrent la dénomination de sans papier. Dans les deux cas, l'objectif est d'obtenir des papiers et l'illégalité est perçue comme un risque à prendre qu'il s'agit de réduire ou de maîtriser.

Devenir harraga ou clandestin est une voie parmi d'autres pour s'enrichir ou survivre mais surtout pour faire quelque chose de sa vie. La clandestinité n'est pas une fin en soi mais une période, un passage dans la vie imposé par les circonstances. Non pas tant hors la loi que malgré la loi. Ainsi, dans leurs parcours les harragas peuvent basculer d'une stratégie légale à une autre illégale, et se percevoir comme "des guerriers », qui ont "appris le droit » et ses limites, et sont devenus "des caméléons ». Aziz, un jeune harraga originaire d'Alger, s'est présenté ainsi : «Les immigrés, ceux qui ont des papiers, nous appellent les blédards, les clandos. Mais nous on n'est pas des clandos, c'étaient les premiers, nous on a appris le droit, la demande d'asile. On sait comment contourner, aller dans le sens inverse des autres, ouvrir des voies. Il y a toujours une solution pour passer ». Les lois et les pratiques limitant dans leurs cas les possibilités de discussion et de négociation ne leur laissent pas beaucoup d'autres choix que le déplacement vers l'illégalité qui, face aux circonstances, se trouve si ce n’est légitimé au moins légitime.

Le harraga est pour les Algériens une figure emblématique de la clandestinité à laquelle tous ne souhaitent pas être assimilés et dont ils se rapprochent dangereusement quand ils ont épuisé toutes les demandes de statuts et essuyé tous les refus. Mais ils préfèrent se dire sans papier. Ils ont le sentiment de ne faire qu'une entorse à la loi, font tout pour garder une couverture légale à leur présence et souhaitent au plus vite sortir

8 Le harraga que mes interlocuteurs traduisent par le terme de grilleur (en référence au harraga brûleur de frontières, mais élargi à toute situation d'irrégularité telle que l'achat de visa, de faux papiers, ou de séjour irrégulier) est le clandestin proche du harraga marocain décrit par Mohamed Nadif (2002). 
de l'illégalité, régulariser leur situation. Ils se définissent plus comme « en situation irrégulière » que comme clandestins. "Pour nous, l'important, c'est les papiers, pas pour vous. C'est rien, ça veut rien dire, c'est pas des papiers qui vont te changer, te donner une identité mais ils ouvrent le chemin. Nous on s'en fout de se revendiquer Algériens, on veut les papiers, du travail, que la France nous accepte, nous accueille tout en restant Algériens » m'a dit Djamel, un adulte de 45 ans. Il m'a expliqué ainsi sa ligne de conduite : "je joue tous leurs jeux pour mieux jouer le mien ».

\section{RÉUSSIR SA VIE AU RISQUE DE L'ILLÉGALITÉ}

Je me suis alors demandée comment font ces migrants individuellement et collectivement pour à la fois «mériter » des papiers et vivre sans papiers, pour prendre des initiatives avec une marge de manœuvre aussi étroite.

\section{L'épreuve de la vie sans papiers : le squat et le travail}

Le logement et le travail sont deux domaines vitaux pour la survie hors du cadre légal. Répondre à ces besoins demande aux « irréguliers » d'élargir leurs réseaux, de mobiliser des liens et de rechercher des ressources nouvelles. Ainsi en a-t-il été de l'occupation d'un squat par une douzaine d'Algériens en situation irrégulière pendant un an, occupation qui leur a permis de prendre pied dans la région et de trouver de nouvelles solutions. Négocié avec un propriétaire immobilier, il a donné lieu à une conjonction d'acteurs. D'un côté, un propriétaire consentant, un policier municipal protecteur, une association qui soutient, une partie des voisins compréhensifs, des conseillers municipaux qui donnent des informations même s'ils réclament le secret. De l'autre, une préfecture qui fait fonctionner ses réseaux d'informateurs et qui fait pression sur les occupants et leurs soutiens ou sur des maires embarrassés. Enfin des squatteurs qui font jouer des rôles à leurs soutiens et s'en servent comme intermédiaires et protecteurs. De même qu'ils utilisent le récépissé de demandeur d'asile comme couverture juridique pour se donner le droit d'être là.

Le squat est ainsi devenu un lieu connu, « reconnu », ayant acquis une certaine visibilité, relativement protégé qui permettait de gagner du temps, d'avoir une stabilité provisoire pour trouver d'autres solutions. Par ailleurs, malgré son inconfort, il a pu devenir un lieu de ressources, de socialisation, de coopération, de captation et de circulation d'informations autour de bien d'autres questions que celles du seul logement. Il a servi de point de départ pour des ancrages dans la ville. Entre temps, un parc de logements de sans papiers arrivés sur la ville à la même période s'est constitué peu à peu où l'on peut observer un jeu de chaises musicales et une rénovation de l'immobilier hors circuit institutionnel. Pour le logement toujours, des accords voient le jour entre familles de sans papiers et retraités qui font des va et vient entre la France et leur pays, entre jeunes sans papiers et jeunes résidents bénéficiant de l'APL. Certains occupants du squat sont ainsi passés de la rue, du foyer, du squat à la co-location, à la sous-location. Quelques-uns, en négociant directement avec les propriétaires, ont réussi à s'installer dans des appartements ou des maisons individuelles qu'ils ont réhabilités. 
Trouver du travail et des revenus est l'objet de construction de parcours et de « carrières » au sens d'Howard Becker (1985) et Everett Hughes (1996). Si au départ trouver du travail est une affaire personnelle qui commence souvent par un recours aux liens familiaux ou d'origine commune, par la suite l'espace de recherche s'agrandit, des organisations et des formes sociales se développent, des compétences professionnelles et relationnelles s'affirment, des périodes se succèdent. Chaque lieu recèle des niches de travail que les migrants s'attachent à repérer. Si les travaux saisonniers agricoles sont les offres d'emploi les plus fréquentes, les migrants tâchent de ne pas se laisser enfermer dans ce secteur d'activités. Les emplois dans la restauration, dans le bâtiment, dans la mécanique, dans la rénovation de logements, dans les services à domicile, la coiffure, la couture, dans la récupération, réparation et revente ou sur les marchés et dans les commerces leur permettent de mobiliser leurs compétences. Le plus souvent leurs relations avec leurs «employeurs » ne se résument pas à de seules relations d'exploitation.

Des carrières professionnelles se construisent sur des compétences antérieures, acquises sur le tas ou encore mises en commun entre migrants et elles demandent un incessant travail relationnel. C'est ainsi que Djamel qui a exercé en Algérie de nombreuses professions est passé de la vente dans une boucherie marocaine à titre gracieux en échange d'un logement, à l'installation de l'électricité sur le chantier d'une école, au déchargement de camions, au dépannage à domicile d'appareils ménagers, au ramassage de fruits, à l'exploitation du camion de frites et sandwichs d'un cousin, à la taille de la vigne, à la taille de haies, à la construction d'un mur en pisé chez un architecte évangéliste, à la maçonnerie, au ramassage de l'ail, à la peinture d'appartements, à la récupération et réparation d'appareils électroménagers et à la rénovation d'un hôtel particulier pour un voisin avec qui il a sympathisé. D'autres font équipe comme Ahmed et son associé Abdel. L'un est mécanicien et l'autre affable et sociable se charge des relations commerciales. Ils trouvent leur clientèle sur un marché et prennent en charge en particulier des voitures des saisonniers agricoles, mais leur réputation s'est étendue au-delà de ce cercle. Ils connaissent bien les casses, les vendeurs de pièces et s'occupent également de la préparation des contrôles techniques. Et ce n'est pas leur seule activité. Les moins compétents rencontrent plus de difficultés mais ils peuvent apprendre et faire équipe.

Cet incessant travail relationnel ne doit pas faire oublier que son résultat se compte en heures, en jours, parfois en semaines de travail mais rarement plus.

\section{Les voies de la régularisation : le mariage}

En dehors des négociations au cas par cas avec les préfets et les administrations, qui aboutissent difficilement, le mariage reste finalement pour les déboutés la seule voie vers la régularisation, voie semée d'embûches, de contraintes et de soupçons. Cependant la « régularisation » par le mariage est souvent l'opération de la dernière chance ; c'est aussi une opération à risques, entre dettes et tromperies. Le conjoint sans papier hésite entre contrôles et contraintes, stratégies et aspirations. Le choix de son conjoint n'est pas tout à fait libre et conduit à changer les rapports aux 
normes. La monnaie à rendre, dette symbolique, argent ou autres services, peut être lourde. Mais, au-delà du fait qu'il puisse être contraint, arrangé, acheté ou blanc, nous pouvons parler de mariages négociés. Des conduites légales peuvent apparaître comme immorales et des conduites illégales peuvent au contraire se révéler plus honnêtes. C'est ainsi parce qu'Ahmed, 26 ans, musulman pratiquant, ne veut commettre aucun péché et redoute au plus haut point d'être en tort vis-à-vis de la loi qu'il se décide à faire un mariage blanc. Il choisit de jouer «la comédie du mariage » avec une jeune fille française prête à lui offrir ce don d'hospitalité pour les deux années nécessaires à l'obtention de ses papiers en $2004^{9}$. Débouté de ses démarches légales, entre les dangers de la loi qui se rapprochent et les propositions pressantes de mariage de la famille qui ne lui conviennent pas, la meilleure solution qui se présente est le mariage blanc. La cérémonie et le projet de vie commune de deux ou trois ans ont été négociés dans le détail entre les deux conjoints, avec leurs familles respectives, mais aussi avec leurs alliés et partenaires : un médecin, des élus, des membres d'associations, des amis et le parrain, un bénévole catholique. Certains ont été informés de la nature de ce mariage et d'autres non. Il faut à la fois que cela se passe bien selon les normes légales et selon les normes morales et religieuses. Pour tromper la loi mais aussi pour que ce soit viable. Dans ce cas le mariage blanc, bien plus qu'un détournement de la loi, un mensonge, une comédie, implique une véritable expérience, l'invention d'une manière de vivre. Il est le résultat des compétences relationnelles d'Ahmed. Il se donne à lui et aux autres le sentiment qu'il reste en partie maître de ses choix. Le contrat rempli, le couple temporaire s'est séparé. Avec ses papiers, Ahmed peut envisager dans d'autres conditions un «vrai » mariage qu'il n'aurait jamais pu espérer avant. Auprès de ses intimes il évoque le mariage avec une fille du bled.

\section{Les retours}

La perspective qui devient commune aux sans papiers est de parvenir à une régularisation pour envisager librement la possibilité d'un retour. Un tournant décisif, une étape importante dans le parcours et la quête de reconnaissance. La réussite se joue aussi là-bas et le retour permet une forme de reconnaissance réciproque. Les sans papiers expriment à la fois la hantise du retour au bled prématuré par l'expulsion en même temps que l'attente et l'espoir d'un retour une fois régularisés. Un acte significatif que beaucoup entreprennent le plus vite possible une fois régularisés et pour certains de façon répétée, savourant le plaisir tant espéré et tant attendu d'aller et venir. De retour, les régularisés ramènent des cadeaux mais ils savent que la valeur première qu'ils rapportent ce sont leurs papiers et les possibilités de circulation, la liberté qu'ils leur accordent. Ils savent de part et d'autre de la Méditerranée qu'ils ne sont pas tant allés au paradis que partis refaire leur vie. L'accompagnement en juin 2005 du retour de Malika, partie quatre ans auparavant, m'a permis d'approcher les différentes dimensions et significations de ce temps.

9 Depuis la loi Ceseda adoptée en 2006, la durée de vie commune pour demander un titre de séjour de dix ans a été portée à trois ans. 
Malika, partie après un divorce, revient surtout pour revoir son fils et renouer les liens. Mais ses retours ce sont aussi le retour chez son père, dans la belle famille, auprès de ses collègues de travail de la daïra ${ }^{10}$, de ses copines au cours d'une soirée, dans sa famille à l'occasion de la fête du brevet des neveux, au hammam, à la plage et dans les commerces pour retrouver les plaisirs du quotidien au bled. Il lui a fallu retrouver sa place, régulariser les liens avec sa famille et renégocier les relations avec la belle-famille, signaler ses bonnes intentions, regagner l'estime et la confiance pour se réconcilier. Mais le parcours de Malika est aussi jugé, apprécié comme celui d'une femme autonome qui a su prendre une décision, faire un choix. Ses collègues de travail fêtent le retour d'une amie et d'une héroïne et me disent à propos de son divorce et de son départ : "Malika, elle est forte de caractère, elle a de la personnalité... Partir c'est une question de choix de vie mais faut l'assumer. Si, ici, t'es pas capable d'imposer tes choix, de divorcer, tu n'y arriveras pas plus à l'étranger... ». Ses amies qui la reçoivent une soirée n'ont pas envie de partir mais admirent Malika qui a réussi à refaire sa vie et a trouvé un amoureux. "Tu as réussi à refaire ta vie, tu es bien, c'est bien ». Lors d'une fête familiale, celle qui s'est absentée doit retrouver sa place, faire bonne figure. On reconnaît qu'elle a réussi son entreprise mais l'expérience qui lui a permis d'y parvenir est gommée. À cette impression de silence sur son vécu en France de « sans papière » Malika m'a répondu : «..., ils s'en foutent, ils veulent pas savoir. Ce qu'ils voient c'est Hamdoullah, t'as fait tes papiers. Tu es arrivée à ton but. Ce que tu as souffert, ils veulent pas l'entendre. Pour eux, c'est comme si tu étais partie hier. Le passage en France, sans papiers, c'est comme si ça n' existait pas. Ils te regardent, te disent tu as l'air bien, heureuse. Et c'est les premiers à te mettre dans les pattes un neveu, une nièce pour que tu l'emmènes en France».

Les premiers retours après la régularisation ne marquent pas la fin, le but ultime de la migration mais ils constituent un moment, une étape à mi-parcours propice aux bilans et aux projets. Qu'est ce qui a été gagné, perdu, qu'est ce qui a changé ? Qu'est ce qu'il faut en dire, en faire ? Comment poursuivre, rebondir, continuer à vivre ici, là-bas, ici et là-bas ?

\section{CONCLUSION : UNE MIGRATION CHOISIE MAIS ILLÉGALE}

Quel est le projet migratoire illégal des sans papiers ? Ce projet peut varier selon leurs conditions de vie, leur niveau d'instruction, leur âge, leur situation matrimoniale, leurs chances de régularisation. Il vise un objectif qui se décline selon plusieurs modalités : parvenir par soi même à une réussite individuelle socialement reconnue.

Cette migration serait envisagée comme un moyen d'obtenir des droits et des libertés, des revenus et des biens. "Faire ses papiers et de l'argent » pour être reconnu comme un individu capable d'agir et de réussir sa vie, malgré les contraintes juridiques

10 La daïra est la sous-préfecture. 
mises en place par les États européens. La circulation apparaît alors comme une façon de se constituer comme sujet et l'illégalité comme un risque à prendre que les migrants tentent de réduire ou de maitriser.

Il est question de faire ou refaire sa vie. Sans rien devoir à personne, parce qu'on ne peut plus ou qu'on ne veut plus compter sur les autres. Mais le retour est au programme pour que leur réussite soit socialement reconnue par leurs proches. Revenir pour montrer ses papiers, ses richesses, gagner le respect et l'estime, être reconnu comme un être humain qui a de la valeur, devenir quelqu'un dans le regard des autres. Sortir de la condition de celui que personne ne regarde parce qu'il n'a pas de travail, pas de logement, pas de famille, pas d'argent, pas de biens. C'est aussi remplir son rôle de parent en assurant un meilleur avenir pour ses enfants.

La circulation pour se constituer comme sujet cela commence par bouger, être capable de partir, de tout quitter, de prendre le risque de l'aventure, du voyage, d'obtenir un visa, de passer la frontière, de vivre ailleurs, même sans papiers. Cela continue par le fait de donner de ses nouvelles en téléphonant, en envoyant de l'argent, des colis, des photos, en donnant des informations et en servant de relais pour ceux qui veulent partir. Une entreprise couronnée de succès et de prestige quand vient le retour avec des papiers et si possible une voiture, des euros et des biens. L'apothéose est atteinte quand l'argent gagné là-bas peut être réinvesti dans une maison, un commerce ou une affaire. L'échec, la honte suprême, c'est le retour forcé faisant suite à une expulsion, qui s'alourdit du soupçon que l'expulsé a dû mal se comporter pour mériter une telle sanction.

«Faire ses papiers » est le sésame pour réussir, se projeter dans l'avenir et envisager le retour. Plus les années sans papiers passent, plus le moment du retour est attendu, espéré. Les papiers représentent donc d'abord le droit de circuler. Ils sont aussi le symbole du choix. Celui qui a des papiers peut choisir où il va, où il vit, avec qui il se marie, où il travaille, où il habite, même si c'est relatif. Plus qu'un choix de résidence ou de nationalité, c'est la clef qui ouvre le chemin et élargit les possibilités. Les papiers représentent aussi l'accès à des droits sociaux qui apportent un peu de sécurité, un autre rapport au temps. Enfin, ils donnent une identité. Non pas une identité nationale mais celle d'un être humain qui a des droits. 


\section{Références bibliographiques}

BATTEGAY Alain (1996) Le migrant acteur, la migration comme activité, in M. Péraldi et E. Perrin (dir.), Réseaux productifs et territoires urbains, Presses Universitaires du Mirail, $386 \mathrm{p}$.

BECKER Howard (1985) Outsiders, Métaillé, Paris, 247 p.

BRACHET Olivier (1997) L'impossible organigramme de l'asile en France. Le développement de l'asile au noir, Revue européenne des migrations internationales, vol. 13, $\mathrm{n}^{\circ}$ 2, pp. 7-35.

BRUN François (2006) Les sans papiers : affaire d'humanité ou question politique ? Migrations société, vol. $18, \mathrm{n}^{\circ} 104$, pp. 103-106.

DJERBAL Daho (2001) La démocratisation impossible, Transeuropéennes, $\mathrm{n}^{\circ} 21$, http://transeuropeennes.gaya.fr/revue/revue.html

FRANGUIADAKIS Spyros, JAILLARDON Edith, BELKIS Dominique (2005) En quête d'asile, aide associative et accès au(x) droit(s), éditions LGDJ, $287 \mathrm{p}$.

FRIGOLI Gilles (2004) Le demandeur d'asile : un exclu parmi d'autres ? Revue européenne des migrations internationales, vol. 20, $\mathrm{n}^{\circ} 2$, pp. 153-165.

HUGHES Everett C. (1996) Le regard sociologique. Essais choisis, textes rassemblés et présentés par Jean-Michel Chapoulie, Paris, École des Hautes Études en Sciences Sociales, $344 \mathrm{p}$.

LEGOUX Luc (1999) La demande d'asile, in Dewitte Philippe (dir.), Immigration et intégration, l'état des savoirs, Paris, La Découverte, pp. 341-351.

MARIN Luca (2006) Gêner pour exister, Migrations Société, vol. 18, n 104, CIEMI, pp. 121147.

MISSAOUI Lamia et TARRIUS Alain (2006) Villes et migrants, du lieu-monde au lieu-passage, Revue européenne des migrations internationales, vol. $22, \mathrm{n}^{\circ} 2$, pp. 43-65.

MORICE Alain (2004) Le travail sans le travailleur, Plein Droit, $\mathrm{n}^{\circ}$ 61, pp. 2-7.

MUSETTE Mohamed Saïb (ed.) (2004) Sociologie de la jeunesse : regards critiques sur les jeunes et la santé en Algérie, Centre de recherche en économie appliquée pour le développement (CREAD), Alger.

NADIF Mohamed (2002) La culture du hrigue, le mirage de la réussite sociale et l'obsession de l'immigration, in Informations et commentaires, Le goût amer de nos fruits et légumes, $\mathrm{n}^{\circ}$ hors série, Forum civique européen, pp.93-98.

REA Andréa (2002) Le travail des sans papiers et la citoyenneté domestique, in M. Péraldi (dir.), La fin des norias? Réseaux migrants dans les économies marchandes en Méditerranée, Paris, Maisonneuve et Larose, pp. 459-478.

SIMEANT Johanna (1998) La cause des sans papiers, Paris, Presses de la Fondation nationale des sciences politiques, $504 \mathrm{p}$.

STRAUSS Anselme (1999) La trame de la négociation, textes réunis et présentés par Isabelle Baszanger, L'Harmattan, $311 \mathrm{p}$.

TARRIUS Alain (2001) Les nouveaux cosmopolitismes, L'Aube, 267 p.

TERRAY Emmanuel (1999) Le travail des étrangers en situation irrégulière ou la délocalisation sur place, in Balibar, Chemillier-Gendreau, Costa-Lascoux, Terray, Sans papiers : l'archaïsme fatal, Éditions La Découverte, pp. 9-34.

TÊTU Marie-Thérèse (2008) Vivre sans papiers et «mériter» ses papiers. Usages de la loi, pratiques illégales et reconnaissance, in Payet J.-P. et Battegay A. (ed.), La reconnaissance à l'épreuve, explorations socio anthropologiques, éditions Peter Lang, Genève, pp. 227-235.

TÊTU Marie-Thérèse (2007) L'énigme des sans papiers algériens en France. De la conversation à l'ethnographie multi sites, Anthropologie et Sociétés, n 2, vol. 31, Montréal, pp. 267-285.

TÊTU Marie-Thérèse (à paraître 2009) Clandestins au pays des papiers. Une expérience auprès des migrants algériens, La Découverte et le CIEMI. 
WALDINGER Roger (2006) Transnationalisme des immigrants et présence du passé, Revue européenne des migrations internationales, vol. $22, \mathrm{n}^{\circ} 2$, pp. 23-41.

WEIL Patrick (1991) La France et ses étrangers. L'aventure d'une politique de l'immigration 1939-1991, Calmann Lévy, Paris, 403 p.

WEIL Patrick (2002) Qu'est ce qu'un Français ? Histoire de la nationalité française depuis la Révolution, Grasset, Paris, $401 \mathrm{p}$.

WIHTOL DE WENDEN Catherine (1999) Faut-il ouvrir les frontières?, Presses de la fondation de sciences politiques, Paris, $120 \mathrm{p}$. 


\title{
La migration au risque de l'illégalité, entre France et Algérie (1998-2004)
}

\author{
Marie-Thérèse TÊTU
}

\begin{abstract}
À partir d'une approche socio anthropologique, cet article se propose de rendre compte du phénomène des migrations irrégulières algériennes vers la France en se plaçant du point de vue des migrants. L'intention n'est ni de nier, ni de survaloriser les circulations à l'initiative des migrants mais d'examiner leurs formes et leurs sens dans ce cas. Nous avons mené nos investigations et développé nos analyses dans trois directions qui apparaissaient essentielles dans leur expérience : la carrière administrative et ses démarches, la vie ordinaire et ses épreuves, les liens avec l'Algérie. Au croisement de ces trois sources, les matériaux constitués et recueillis permettent de dessiner les profils et les figures de cette migration. Au fil des parcours suivis, les épreuves de la vie sans papiers, la négociation d'un mariage comme voie de régularisation et le moment du retour deviennent autant d'étapes d'un projet migratoire en quête de reconnaissance et de réussite sociale ici et là-bas.
\end{abstract}

\section{The Migration at the Illegality's Risk, between France and Algeria (1998-2004)}

\author{
Marie-Thérèse TÊTU
}

From a socio anthropological approach, this article suggests to report the phenomenon of the Algerian irregular migrations in transit to France, by the migrants point view. The intention is neither to deny nor to overvalue the migrant circulation but examining their forms and their directions in that case. We made our investigations and evolved our analyses in three directions which seemed essential in their experience: the administrative career and its approach, the plain life and its tests, the links with Algeria. At the crossroad of these three sources, the constituted and gathered information allow to draw the profiles and the features of this migration. During the followed routes, the life tests of paperless, the marriage negotiation as a regularization way and the return moment, become so many stages of a migratory project to gain recognition and social success here and over there.

\section{La migración, a riesgo de la ilegalidad, entre Francia y Argelia (1998-2004)}

\section{Marie-Thérèse TÊTU}

A partir de una aproximación socio-antropológico, este artículo se propone dar cuenta, del fenómeno de las migraciones irregulares argelinas hacia Francia, desde el punto de vista de los emigrantes. En este caso, la intención no es negar, ni sobrevalorar la circulación por iniciativa de los emigrantes, sino examinar sus formas y sus direcciones. Hemos llevado nuestras investigaciones y desarrollado nuestros análisis en tres direcciones que parecían esenciales en sus experiencias: La carrera administrativa y sus gestiones, la vida ordinaria y sus pruebas y los lazos con Argelia. La unión de estos tres enfoques, las fuentes, constituidas por los materiales recogidos, nos permiten dibujar los perfiles y las figuras de esta, migración. En el transcurso de sus recorridos, las pruebas de una vida sin papeles, la negociación de un matrimonio como vía de regularización y el momento de la vuelta, se hacen tantas etapas de un proyecto migratorio en busca de reconocimiento y de logros sociales aquí y allá. 